\title{
Addressing the management of atrial fibrillation - a systematic review of the role of dronedarone
}

This article was published in the following Dove Press journal:

International Journal of General Medicine

25 May 2012

Number of times this article has been viewed

\section{Gian Marco Podda' \\ Giovanni Casazza ${ }^{2}$ \\ Francesco Casella ${ }^{3}$ \\ Franca Dipaola ${ }^{4}$ \\ Emanuela Scannella ${ }^{3}$ \\ Ludovica Tagliabue ${ }^{5}$}

'Medicina III, Department of Medicine, Surgery and Dentistry, San Paolo Hospital, University of Milan, Milan, Italy; ${ }^{2}$ Department of Clinical Science,

"L. Sacco", University of Milan,

Milan, Italy; ${ }^{3}$ Department of Internal

Medicine, L. Sacco Hospital, Milan,

Italy; ${ }^{4}$ Internal Medicine, Istituti

Clinici di Perfezionamento Sesto San

Giovanni, Italy; ${ }^{5}$ School of Hygiene and

Preventative Medicine, University of

Milan, Milan, Italy
Correspondence: Gian Marco Podda Medicina III, San Paolo Hospital,

University of Milan, Milan, Italy

Tel +390281844522

Fax +390250323089

Email gmpodda@gmail.com
Background: Atrial fibrillation (AF) is the most common sustained arrhythmia. It occurs in $1 \%-2 \%$ of the general population and its prevalence increases with age. Dronedarone, a noniodinated benzofuran similar to amiodarone, was developed as an antiarrhythmic agent for patients with atrial fibrillation. The aim of our systematic review was to critically evaluate randomized controlled trials that compared treatment with dronedarone versus placebo or amiodarone in patients with atrial fibrillation.

Methods: Electronic databases (MEDLINE, Embase, and Central) were searched up to November 2011 with no language restrictions. We included randomized controlled trials in which dronedarone was compared to placebo or other drugs in patients with AF. Internal and external validity was assessed.

Results: We identified seven papers corresponding to eight randomized controlled trials. The DAFNE, EURIDIS/ADONIS, and ATHENA trials demonstrated a reduction of AF recurrence with dronedarone as compared to placebo in patients with nonpermanent AF. The DIONYSOS study showed that dronedarone is less effective for the prevention of recurrent AF but improved tolerability as compared to amiodarone. Considering patients with permanent AF, the ERATO trial showed that dronedarone had rate-control effects while the PALLAS study was stopped early since stroke, myocardial infarction, systemic embolism, or death from cardiovascular causes were significantly more frequent in subjects treated with dronedarone as compared to placebo. The ANDROMEDA trial included patients with recent hospitalization for heart failure and was terminated early because of excess of deaths in the dronedarone group.

Conclusion: Like most antiarrhythmic drugs, dronedarone reduces the recurrence of AF in patients with paroxysmal or persistent AF as compared to placebo. However, relapse rates in the first year of therapy are high. Moreover, dronedarone showed to be less effective than amiodarone. Finally, dronedarone should be avoided in patients with permanent AF and a high risk for cardiovascular events or severe congestive heart failure.

Keywords: amiodarone, arrhythmia, cardiovascular events

\section{Introduction}

A 68-year-old man presented to the emergency department for a sudden onset of palpitation, fatigue, and dyspnea on exertion. FC was the medical doctor on duty. The patient denied chest pain and reported a long-standing history of hypertension, but there was no history of coronary artery disease and no other cardiovascular risk factors. Home medications were bisoprolol ( $5 \mathrm{mg}$ daily) and enalapril (20 mg daily).

Twelve lead-ECG showed atrial fibrillation (AF) with a ventricular rate of $160 \mathrm{bpm}$; blood tests were unremarkable. The patient was treated with intravenous propafenone 
with successful cardioversion to sinus rhythm. Considering the risk factors for cardioembolic stroke (hypertension and age determining a CHA2D2-VASC score of 2 points), ${ }^{1}$ oral anticoagulant therapy was started.

Over the previous months the patient had had two emergency department visits for palpitation and dyspnea on exertion due to AF with high ventricular rate; in both cases he had spontaneous cardioversion to sinus rhythm in the emergency department. After the first episode of AF, a transthoracic echocardiogram was performed that showed moderate increased left ventricular wall thickness (16 mm), normal left ventricular and atrial sizes, and normal systolic function. Thyroid function tests were normal.

Considering the disabling symptoms during the recurrences of $\mathrm{AF}$ and the inability to attain adequate rate control, FC decided to adopt a rhythm control strategy for the maintenance of sinus rhythm. Which antiarrhythmic treatment should be started in a patient with AF and left ventricular hypertrophy without coronary artery disease?

In order to choose the appropriate therapy, FC decided to look at two of the most authoritative clinical guidelines on the topic, edited respectively by the American College of Cardiology Foundation/American Heart Association (ACCF/AHA $)^{2,3}$ and by the European Society of Cardiology (ESC). ${ }^{4}$ As the recommendations were discordant, FC was puzzled. In fact, while ACCF/AHA suggested amiodarone as first-line treatment in this clinical setting, ESC guidelines recommended dronedarone (Table 1).

So, FC wondered: "Was dronedarone actually useful to maintain sinus rhythm? Compared to amiodarone, was dronedarone more efficacious and safe? What was the available evidence? Did any study evaluate clinical relevant end points such as mortality?"
$\mathrm{AF}$ is the most common sustained arrhythmia. It occurs in $1 \%-2 \%$ of the general population and its prevalence increases with age. ${ }^{5,6}$ It may cause disabling symptoms, with impairment of both functional status and quality of life.

The ACC/AHA ${ }^{2,3}$ and $\mathrm{ESC}^{4}$ guidelines distinguish four types of AF: (1) "first detected or diagnosed", (2) paroxysmal, (3) persistent, and (4) permanent AF. "First detected or diagnosed AF" is AF identified for the first time, independently of its duration or the presence of symptoms. Paroxysmal AF is defined as recurrent AF that terminates spontaneously in less than 7 days, usually in less than 48 hours. Persistent AF is AF lasting longer than 7 days or requiring pharmacological or electrical cardioversion to sinus rhythm. Finally, permanent AF lasts more than 1 year. Moreover, into the category of persistent AF, ESC distinguishes a "long-standing persistent AF" with 1 year or more of duration after the adoption of a rhythm-control strategy. For permanent AF, on the contrary, rhythm-control strategy is not pursued.

AF is associated with increased morbidity and mortality, mainly due to stroke and heart failure. ${ }^{7,8}$

Management of AF patients is focused on reducing symptoms and preventing the complications associated with arrhythmia. Two therapeutic strategies can be identified: a "rhythm-control" option, when antiarrhythmic drugs, with or without electrical cardioversion, are used to restore sinus rhythm, and a "rate-control" strategy, the main objective of which is to control ventricular response rate. In addition, stroke prophylaxis therapy is required in most AF patients and antithrombotic therapy is prescribed according to stroke risk stratification schemes, such as CHA2D2-VASC, ${ }^{1}$ taking into account also the patient's risk of bleeding.

To date, randomized controlled trials have failed to demonstrate the superiority of one strategy over the other. ${ }^{9-11}$

Table I Choice of antiarrhythmic drug according to underlying pathology: comparison of ACCF/AHA ${ }^{2,3}$ and ESC ${ }^{4}$ guidelines

\begin{tabular}{lll}
\hline Underlying pathology & ACCF/AHA & ESC \\
\hline $\begin{array}{l}\text { Minimal or no heart disease } \\
\text { Hypertension }\end{array}$ & Dronedarone flecainide propafenone sotalol & Dronedarone flecainide propafenone sotalol \\
With LVH & Amiodarone & Dronedarone \\
Without LVH & Dronedarone flecainide propafenone sotalol & Dronedarone flecainide propafenone sotalol \\
CAD & Dronedarone sotalol dofetilide & Dronedarone sotalol \\
Heart failure & & \\
Stable NYHA I/II & Amiodarone dofetilide & Dronedarone \\
NYHA III/IV or unstable NYHA II & Amiodarone dofetilide & Amiodarone \\
\hline
\end{tabular}

Notes: In the congestive heart failure category, ACCF/AHA has two different subcategories: stable NYHA I/II and NYHA III/IV or unstable NYHA II. Unstable is defined as cardiac decompensation within the prior 4 weeks.

Abbreviations: ACCF/AHA, American College of Cardiology Foundation/American Heart Association; ESC, European Society of Cardiology; LVH, left ventricular hypertrophy; CAD, coronary artery disease; NYHA, New York Heart Association. 
Many drugs have been employed as antiarrhythmic options: amiodarone seems to be the most effective in preventing recurrences of $\mathrm{AF},{ }^{12,13}$ but its use is limited by toxicity. ${ }^{14}$

Dronedarone, a noniodinated benzofuran similar to amiodarone, was developed as an antiarrhythmic agent for patients with AF. Because of the structural differences between amiodarone and dronedarone, particularly the deletion of iodine molecules, dronedarone has been supposed to have similar efficacy to amiodarone but fewer thyroid and pulmonary side effects.

This systematic review looked at the randomized controlled trials (RCTs) conducted to date that compared treatment with dronedarone versus placebo or amiodarone in patients with AF. Our aim was to assess the efficacy and side-effects of dronedarone and to critically evaluate current evidence.

\section{Methods}

\section{Eligibility criteria}

A bibliographic search was performed starting from the acronym PICOS (Patients or target population included in the trials, Intervention and Comparison, Outcome(s) assessed, type of Studies selected), suggested for formulating relevant and precise questions that can be answered through research and literature sources. ${ }^{15}$ It was decided to look for only RCTs, as they are considered to be at the first level of evidence in determining the benefits of a treatment. ${ }^{16}$

PICOS was defined in the following way:

- Types of patients: Participants of any age and gender affected by AF;

- Types of intervention and comparison: dronedarone at any dose versus placebo or other antiarrhythmic drug;

- Types of outcome measures: No restriction. Our aim was to exhaustively evaluate dronedarone's efficacy and safety;

- Types of study: RCT.

\section{Information sources and search criteria}

RCTs were identified by searching electronic databases (Embase, PubMed, and Central). No limit was applied for language or for year of publication of the studies. The following search terms to search all trial registers and databases were used: "atrial fibrillation" (participants), "dronedarone" OR “amiodarone/ analogs and derivatives" OR “SR33589” (intervention/control), "randomized controlled trials" (type of study). The last search was run on November 25, 2011. In addition, the reference lists of retrieved studies, recent guidelines, meta-analyses, and other reviews of AF were checked.

\section{Study selection}

Eligibility assessment was performed independently in a blinded standardized manner by two reviewers (FD, ES). Disagreements between reviewers were resolved by consensus, eventually involving a third reviewer (LT).

\section{Data collection process and data items}

For randomized trials a data extraction sheet was developed. Couples of authors (LT and ES, FC and FD, GMP, and GC) independently extracted the information from included studies. Disagreements were resolved by discussion between the two reviewers; if no agreement could be reached, it was planned that a third author (GC or GMP) would resolve controversies.

From each included trial we extracted information regarding: (1) the characteristics of the trial's participants, with particular attention to the percentage of patients with AF at enrollment and inclusion and exclusion criteria in the trial; (2) type of interventions used (dose, duration, and frequency of dronedarone, characteristics of placebo or other antiarrhythmic drugs or registration that no treatment was given as control); (3) type of outcome measures (including length of follow-up and type of analysis, ie, "intention to treat" or "per protocol").

\section{Internal validity - risk of bias}

In order to evaluate the internal validity of the RCTs included, risk of bias was assessed in the following items: (1) generation of allocation sequence; (2) appropriateness of allocation concealment; (3) completeness of outcomes data and (4) outcomes reporting. They were classified as adequate (low risk of bias), inadequate (high risk of bias), or unclear (if no information was reported), following the criteria specified in The Cochrane Handbook of Review of Interventions. ${ }^{17}$ Two reviewers (ES, LT) independently assessed trials according to the predefined quality criteria. Any disagreement was resolved by consulting a third party (GMP).

\section{External validity of the evidence - directness}

Reliable judgments about the external validity of RCTs are essential if treatments are to be used correctly in as many patients as possible in routine clinical practice. The Grading of Recommendations Assessment, Development and Evaluation Working Group, in the rating of the quality of evidence, refers to this aspect as the directness/indirectness of evidence. Directly quoting: "direct evidence comes from research that directly compares the interventions in which we are interested when applied to the populations in which 
we are interested and measures outcomes important to patients". ${ }^{18}$

For each study retrieved, it was decided to analyze the level of directness/indirectness and the external validity of the evidence evaluating some of the items proposed by Rothwell. ${ }^{19}$ Mostly: (1) the differences between the population in the usual clinical setting (general medicine or cardiology division) and those that participated in the study, paying attention to the inclusion and exclusion criteria of the patients and those effectively included in the trial; (2) the differences between the interventions (experimental and comparison) in the study and those used in current practice, such as the type and the dose of the comparator drug and the limitations on the use of nontrial treatments (co-therapy); (3) the problems in outcome measures, as in the utilization of "surrogate" outcomes or, in the case of the use of composite outcomes, the risk linked to every component of the outcome; (4) the adequacy of the length of the follow-up in the trial.

\section{Results}

\section{Study selection}

Figure 1 presents a flow diagram to summarize the study selection process. Our literature search identified
300 references; among these there were no duplicates. Two hundred and eighty-eight records were excluded on the basis of titles and abstracts, twelve of these citations proved to be eligible for inclusion and their full texts were analysed in more detail. Finally, we excluded five studies because they were post hoc analyses of eligible studies and included seven papers corresponding to eight RCTs. ${ }^{20-26}$

\section{Study characteristics}

The main features of the trials included are summarized in Table 2. Table 3 reports the principal characteristics of the participants in each trial.

We decided to group the studies according to clinical setting:

1. RCTs including patients with nonpermanent AF (paroxysmal or persistent);

2. RCTs including patients with permanent AF;

3. RCTs considering patients with heart failure.

Only one study was designed to compare dronedarone and amiodarone for the maintenance of sinus rhythm in patients with nonpermanent $\mathrm{AF}^{23}$

Figure 2 gives a summary of the risk of bias for each study. The overall quality of the evidence from the trials,

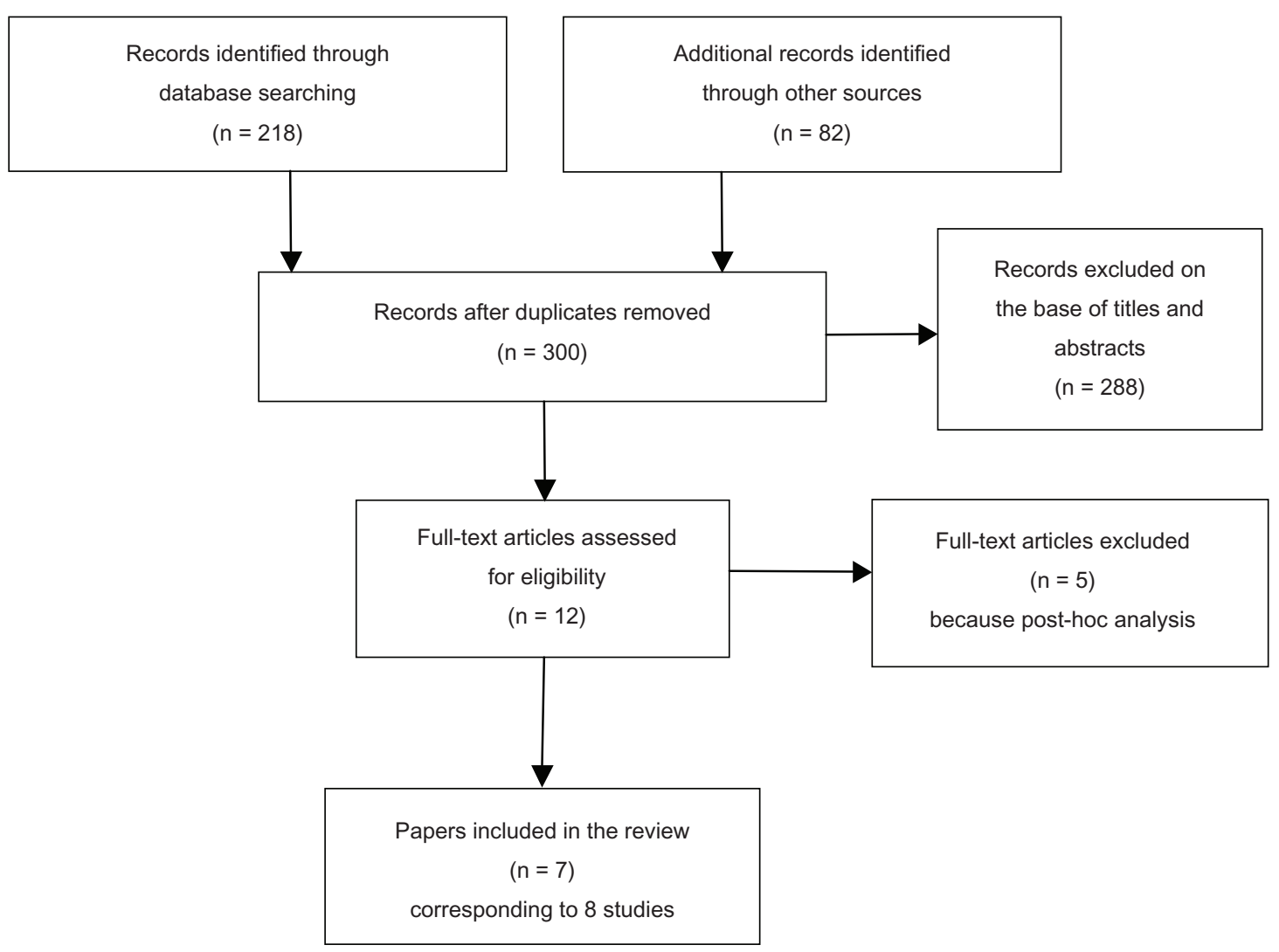

Figure I Flow of information search. 
Table 2 Principal characteristics of studies included

\begin{tabular}{|c|c|c|c|c|c|}
\hline Trial name & $\begin{array}{l}\text { No of patients } \\
\text { (D/C) }\end{array}$ & Dose & Inclusion criteria & $\begin{array}{l}\text { Relevant exclusion } \\
\text { criteria }\end{array}$ & $\begin{array}{l}\text { Mean } \\
\text { follow-up }\end{array}$ \\
\hline DAFNE ${ }^{20, \S}$ & $\begin{array}{l}102 \\
(54 / 48)\end{array}$ & $400 \mathrm{mg}$ bid vs placebo & Persistent AF & $\begin{array}{l}\text { NYHA III-IV; QT > } 500 \mathrm{~ms} ; \\
\text { LVEF }<35 \% \text {; use of other } \\
\text { antiarrhythmic drugs; ICD }\end{array}$ & 6 months \\
\hline $\begin{array}{l}\text { EURIDIS/ } \\
\text { ADONIS }^{21}\end{array}$ & $\begin{array}{l}1237 \\
(828 / 409)\end{array}$ & $400 \mathrm{mg}$ bid vs placebo & $\begin{array}{l}\text { At least one episode of AF in } \\
\text { the last } 3 \text { months; in sinus } \\
\text { rhythm for at least I hour } \\
\text { before randomization }\end{array}$ & $\begin{array}{l}\text { Permanent AF; } \\
\text { HR }<50 \text { beats/min; } \\
\text { NYHA III-IV; } \\
\text { creatinine }>\text { I.7; } \\
\text { use of class I-III } \\
\text { antiarrhythmic drugs }\end{array}$ & 12 months \\
\hline ATHENA $^{22}$ & $\begin{array}{l}4628 \\
(230 \mathrm{I} / 2327)\end{array}$ & $400 \mathrm{mg}$ bid vs placebo & $\begin{array}{l}\text { Paroxysmal or persistent } \\
\text { AF or atrial flutter and at least } \\
\text { one cardiovascular risk factor } \\
\text { (age }>70 \text { years, hypertension } \\
\text { needing at least two drugs, } \\
\text { diabetes, previous stroke, } \\
\text { transient ischemic attack or } \\
\text { systemic embolism, left atrial } \\
\text { diameter }>50 \mathrm{~mm} \text {, LVEF }<40 \% \text { ) }\end{array}$ & $\begin{array}{l}\text { Permanent AF; } \\
\text { HR }<50 \text { beats } / \mathrm{min} ; \\
\text { NYHA IV; GFR }<10 \mathrm{~mL} / \mathrm{min}\end{array}$ & 21 months \\
\hline DIONYSOS ${ }^{23}$ & $\begin{array}{l}504 \\
(249 / 255)\end{array}$ & $\begin{array}{l}400 \mathrm{mg} \text { bid vs } \\
\text { amiodarone } 600 \mathrm{mg} / \text { day } \\
\text { for } 28 \text { days and } \\
\text { then } 200 \mathrm{mg} / \mathrm{day} \\
\text { for } 6 \text { months }\end{array}$ & $\begin{array}{l}\text { Persistent or permanent AF } \\
(>72 \text { hours })\end{array}$ & $\begin{array}{l}\text { Paroxysmal AF; } \\
\text { QT > } 500 \text { ms; NYHA III-IV; } \\
\text { use of class I-III antiarrhythmic } \\
\text { drugs; previous chronic } \\
\text { treatment with amiodarone }\end{array}$ & 6 months* \\
\hline ERATO $^{24}$ & $\begin{array}{l}174 \\
(85 / 89)\end{array}$ & $400 \mathrm{mg}$ bid vs placebo & Permanent AF ( $>6$ months) & $\begin{array}{l}\text { NYHA III-IV; use of other } \\
\text { antiarrhythmic drugs }\end{array}$ & 6 months \\
\hline PALLAS $^{25}$ & $\begin{array}{l}3236 \\
(1619 / 1617)\end{array}$ & $400 \mathrm{mg}$ bid vs placebo & $\begin{array}{l}\text { Permanent AF or atrial flutter } \\
\text { ( }>6 \text { months); age }>65 \text { years } \\
\text { with additional cardiovascular risk } \\
\text { factors (coronary artery disease, } \\
\text { symptomatic heart failure, } \\
\text { LVEF }<40 \% \text {, or a combination of } \\
\text { age }>70 \text {, hypertension, and diabetes) }\end{array}$ & $\begin{array}{l}\text { Non-permanent AF; } \\
\text { HR < } 50 \text { beats } / \mathrm{min} ; \\
\text { QT > } 500 \text { ms; ICD }\end{array}$ & 3.5 months \\
\hline ANDROMEDA $^{26}$ & $\begin{array}{l}627 \\
(310 / 317)\end{array}$ & $400 \mathrm{mg}$ bid vs placebo & $\begin{array}{l}\text { Patients hospitalized with worsening } \\
\text { CHF (NYHA class III or IV) }\end{array}$ & $\begin{array}{l}\mathrm{HR}<50 \text { beats } / \mathrm{min} ; \\
\mathrm{QT}>500 \mathrm{~ms} \text {; use of class I-III } \\
\text { antiarrhythmic drugs }\end{array}$ & 2 months $^{\dagger}$ \\
\hline
\end{tabular}

Notes: §Only the placebo and dronedarone $800 \mathrm{mg}$ daily arms were considered; †'median follow-up; *follow-up duration from 6 months to 22 months.

Abbreviations: D/C, dronedarone/control; AF, atrial fibrillation; NYHA, New York Heart Association; HR, heart rate; LVEF, left ventricular ejection fraction; GFR, glomerular filtration rate; CHF, congestive heart failure; ICD, international cardioverter-defibrillator.

with particular reference to the directness of the evidence produced, is reported for each study.

\section{RCTs including patients in nonpermanent $A F$ DAFNE}

The Dronedarone Atrial FibrillatioN study after Electrical Cardioversion (DAFNE) was a phase II randomized, doseranging study comparing three doses of dronedarone (800, 1200 , and $1600 \mathrm{mg}$ daily) versus placebo in patients with persistent AF (Tables 2 and 3). ${ }^{20}$

In this multicentric study 270 subjects with persistent $\mathrm{AF}$ and for whom cardioversion and antiarrhythmic therapy was warranted were randomized to receive 800,1200 , or
$1600 \mathrm{mg}$ daily doses of dronedarone or placebo. The main analysis was conducted on 151 and 48 patients taking dronedarone and placebo, respectively, after successful pharmacological or electrical cardioversion. Treatment was continued for 6 months. The primary outcome was time to first $\mathrm{AF}$ recurrence; heart rate in case of $\mathrm{AF}$ recurrences, the incidence of side effects and premature study drug discontinuation were secondary end points.

Considering only patients receiving $800 \mathrm{mg}$ of dronedarone daily $(\mathrm{N}=54)$ and placebo $(\mathrm{N}=48)$, time to first recurrence of AF was significantly increased in the dronedarone group (median time of 60 days versus 5.3 days in the placebo arm, relative risk reduction $55 \%$, 95\% confidence interval (CI) 28-72, $P=0.001$; Table 4). 


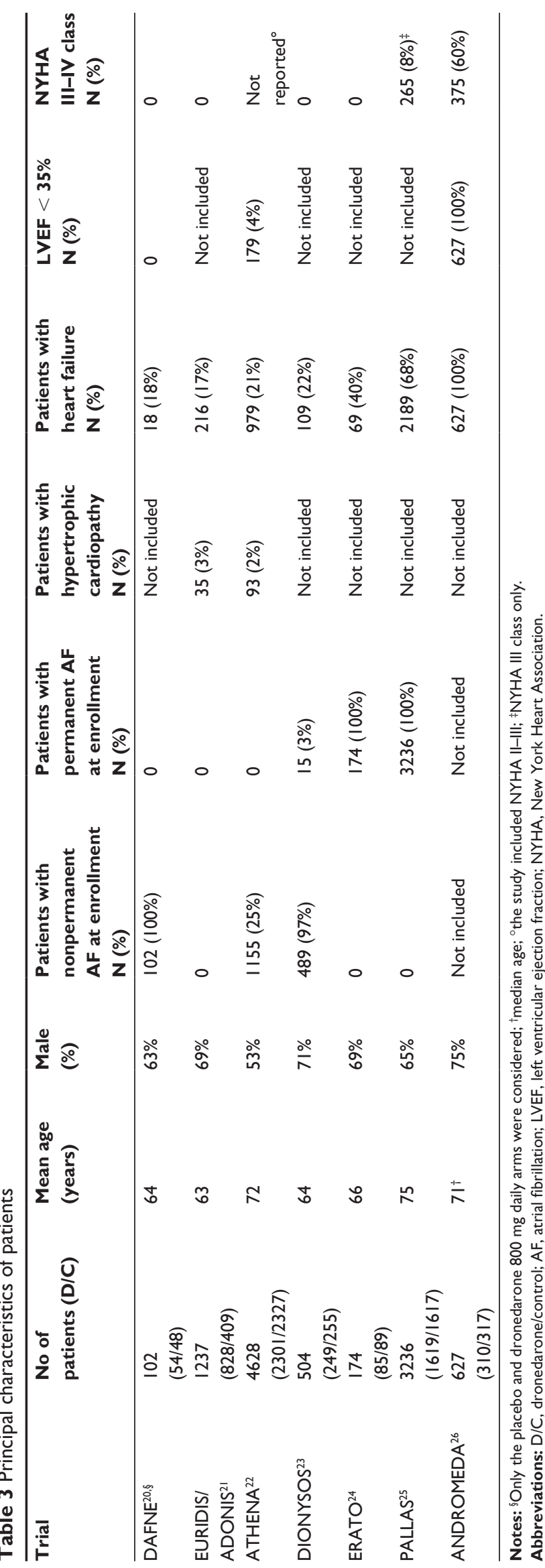

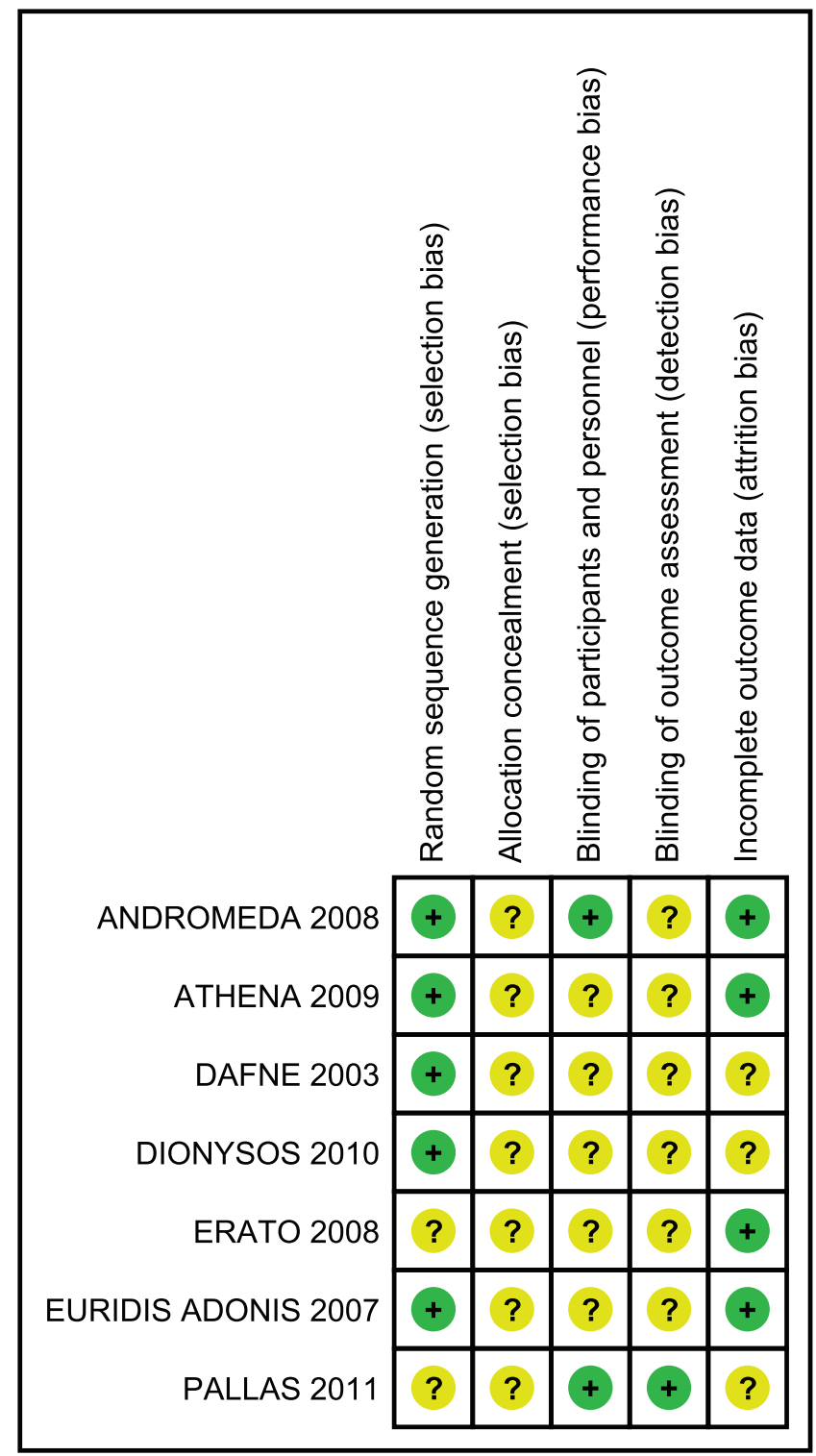

Figure 2 Risk of bias assessment of the RCT included in the review. Legend: green $(+)$, low risk of bias; yellow (?), unknown risk of bias.

Dronedarone was shown to reduce ventricular rate on recurrences of AF in a dose-dependent fashion $(P=0.0001$, value across all doses) indicating some rate-control action. No proarrhythmic effect was reported and no evidence of thyroid, hepatic, neurologic or pulmonary complications was found (Table 5).

\section{Overall quality of the evidence}

The overall risk of bias could not be completely evaluated as much of the information about the methodology was not reported (Figure 2). DAFNE shows that dronedarone may be useful for the maintenance of sinus rhythm after cardioversion. However, several limitations may weaken the quality of the evidence. First of all, none of the end points of 
Table 4 Efficacy end points in dronedarone randomized controlled trials

\begin{tabular}{|c|c|c|c|c|c|}
\hline Trial name & Efficacy end points & Drodenarone & Control & HR (95\% Cl) & $\mathbf{P}$ \\
\hline DAFNE ${ }^{20,8}$ & Time to first recurrence (median of the days) & 60 & 5.3 & Not applicable & 0.001 \\
\hline \multirow[t]{2}{*}{ EURIDIS/ADONIS ${ }^{21}$} & Time to first recurrence (median of the days) & 116 & 53 & Not applicable & Not reported \\
\hline & Recurrence of atrial fibrillation up to 12 months (\%) & $64.1 \%$ & $75.2 \%$ & $0.75(0.65-0.87)$ & $<0.0001$ \\
\hline \multirow[t]{4}{*}{ ATHENA $^{22}$} & $\begin{array}{l}\text { First hospitalization due to cardiovascular events or } \\
\text { death from any cause before hospitalization - N (\%) }\end{array}$ & $734(32 \%)$ & 917 (39\%) & $0.76(0.69-0.84)$ & $<0.001$ \\
\hline & Death from any cause $-\mathrm{N}(\%)$ & $116(5 \%)$ & $139(6 \%)$ & $0.84(0.66-1.08)$ & 0.18 \\
\hline & Death from cardiovascular cause $-\mathrm{N}(\%)$ & $63(3 \%)$ & $90(4 \%)$ & $0.7 \mathrm{I}(0.5 \mathrm{I}-0.98)$ & 0.03 \\
\hline & First hospitalization due to cardiovascular events - N (\%) & $675(29 \%)$ & $859(37 \%)$ & $0.74(0.67-0.82)$ & $<0.001$ \\
\hline DIONYSOS 23 & $\begin{array}{l}\text { First } A F \text { recurrence or premature drug discontinuation } \\
\text { for intolerance or lack of efficacy (efficacy primary } \\
\text { end point) }-N(\%)\end{array}$ & $187(75 \%)$ & $150(59 \%)$ & $1.59(1.28-1.98)$ & $<0.0001$ \\
\hline ERATO $^{24}$ & $\begin{array}{l}\text { Change in mean ventricular rate at the day } 14 \text { day } \\
\text { (beats } / \mathrm{min} \text { ) }\end{array}$ & -11 & +0.7 & Not applicable & $<0.000$ I \\
\hline \multirow[t]{6}{*}{ PALLAS $^{25}$} & $\begin{array}{l}\text { Stroke, myocardial infarction, systemic embolism, } \\
\text { or death from cardiovascular causes - N (\%) }\end{array}$ & $43(3 \%)$ & $19(1 \%)$ & $2.29(1.34-3.94)$ & 0.002 \\
\hline & $\begin{array}{l}\text { Unplanned hospitalization for a cardiovascular cause } \\
\text { or death }-\mathrm{N}(\%)\end{array}$ & $127(8 \%)$ & $67(4 \%)$ & $1.95(1.45-2.62)$ & $<0.001$ \\
\hline & Death for cardiovascular causes $-\mathrm{N}(\%)$ & $21(1 \%)$ & $10(0.6 \%)$ & $2.11(1.00-4.49)$ & 0.046 \\
\hline & Death for any cause - N (\%) & $25(1 \%)$ & $13(0.6 \%)$ & $1.94(0.99-3.79)$ & 0.049 \\
\hline & Stroke - N (\%) & $23(1 \%)$ & $10(0.6 \%)$ & $2.32(1.11-4.48)$ & 0.02 \\
\hline & Hospitalization for heart failure $-\mathrm{N}(\%)$ & $43(3 \%)$ & $24(1 \%)$ & $1.81(1.10-2.99)$ & 0.02 \\
\hline \multirow[t]{5}{*}{ ANDROMEDA $^{26}$} & $\begin{array}{l}\text { Death from any cause or hospitalization for } \\
\text { worsening heart failure }-\mathrm{N}(\%)\end{array}$ & $53(17 \%)$ & $40(13 \%)$ & $1.38(0.92-2.09)$ & 0.12 \\
\hline & Death from all causes $-\mathrm{N}(\%)$ & $25(8 \%)$ & $12(4 \%)$ & $2.13(1.07-4.25)$ & 0.03 \\
\hline & $\begin{array}{l}\text { Hospitalization due to acute cardiovascular } \\
\text { causes }-\mathrm{N}(\%)\end{array}$ & $71(23 \%)$ & $50(16 \%)$ & Not reported & 0.02 \\
\hline & Hospitalization for worsening heart failure - N (\%) & $35(11 \%)$ & $30(10 \%)$ & Not reported & Not reported \\
\hline & Death from arrhythmia or sudden death - N (\%) & $10(3.2 \%)$ & $6(1.9 \%)$ & Not reported & NS \\
\hline
\end{tabular}

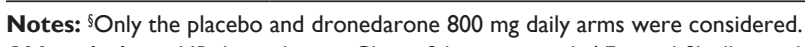

Abbreviations: $\mathrm{HR}$, hazard ratio; $\mathrm{Cl}$, confidence interval; $\mathrm{AF}$, atrial fibrillation; NS, not significant.

the study can be considered clinically relevant or hard end points. Secondly, no data was reported on the incidence of symptomatic AF recurrences, an important issue for patients in rhythm-control strategy. Furthermore, the results of the study were not described in detail as the data of the "intention to treat analysis" on all randomized patients was not shown. Finally, the limited sample size and the short follow-up might have underestimated side effects.

\section{EURIDIS/ADONIS}

The European Trial in Atrial Fibrillation or Flutter Patients Receiving Dronedarone for the Maintenance of Sinus Rhythm (EURIDIS) and the American-Australian Trial with Dronedarone in Atrial Fibrillation or Flutter Patients for the Maintenance of Sinus Rhythm (ADONIS) studies were two phase III, double-blind, randomized trials with the same protocol, that assessed the efficacy of $400 \mathrm{mg}$ of dronedarone

Table 5 Combined main adverse events in dronedarone randomized controlled trials

\begin{tabular}{|c|c|c|c|c|}
\hline \multirow{2}{*}{$\begin{array}{l}\text { Dronedarone versus } \\
\text { placebo }\end{array}$} & \multicolumn{4}{|c|}{ Dronedarone versus amiodarone } \\
\hline & $\begin{array}{l}\text { Dronedarone N/total } \\
(\%)\end{array}$ & $\begin{array}{l}\text { Placebo N/total } \\
(\%)\end{array}$ & $\begin{array}{l}\text { Dronedarone N/total } \\
(\%)\end{array}$ & $\begin{array}{l}\text { Amiodarone N/total } \\
(\%)\end{array}$ \\
\hline Death & I75/5209 (3.3\%) & $167 / 4835$ (3.4\%) & $2 / 249(0.8 \%)$ & $5 / 255(2.0 \%)$ \\
\hline Pulmonary events & $362 / 2752(13.1 \%)$ & $357 / 2795$ (12.8\%) & $0 / 249$ & $0 / 255$ \\
\hline Dysthyroidism & $136 / 3158(4.3 \%)$ & $95 / 2763(3.4 \%)$ & $2 / 249(0.8 \%)$ & I5/255 (5.9\%) \\
\hline Bradyarrhythmia & I70/4733 (3.6\%) & 55/433I (I.3\%) & $47 / 249(18.9 \%)$ & $74 / 255$ (29.0\%) \\
\hline Heart failure ${ }^{\S}$ & $94 / 2823(3.3 \%)$ & $54 / 2343(2.3 \%)$ & $16 / 249(6.4 \%)$ & $19 / 255(7.4 \%)$ \\
\hline Gastrointestinal events & $370 / 2752(13.4 \%)$ & $236 / 2795(8.4 \%)$ & $32 / 249(12.8 \%)$ & I3/255 (5.1\%) \\
\hline Liver toxicity & $173 / 4793(3.6 \%)$ & $98 / 4403(2.2 \%)$ & $30 / 249(12.0 \%)$ & $27 / 255$ (10.6\%) \\
\hline Increased creatinine levels & I85/5043 (3.7\%) & $43 / 4648(0.9 \%)$ & Outcome not considered & Outcome not considered \\
\hline
\end{tabular}

Notes: \#Cough and dyspnea were included; אaccording to the definition of the authors of the trial. 
twice a day in maintaining sinus rhythm in patients with an history of nonpermanent AF or flutter ${ }^{21}$ (Tables 2 and 3). EURIDIS was performed in 12 European countries, while ADONIS was performed in the United States of America, Canada, Argentina, Australia, and South Africa. The two studies enrolled a total of 1237 patients with paroxysmal or persistent AF or flutter; patients were randomized to dronedarone $(\mathrm{N}=828)$ or placebo $(\mathrm{N}=409)$. New York Heart Association (NYHA) class III or IV congestive heart failure patients were excluded. The primary outcome was the time to the first documented AF relapse. The main secondary end points were symptoms related to $\mathrm{AF}$ and the mean ventricular rate during the first recurrence.

Combined results from the two studies are reported in Tables 4 and 5. In the 12-month follow-up period, the two trials showed a longer time to recurrence of the first $\mathrm{AF} /$ atrial flutter in the dronedarone group (median time to first recurrence 116 versus 53 days in the placebo group, $P$-value not reported) and a lower AF recurrence rate up to 12 months (hazard ratio (HR) 0.75 , 95\% CI 0.65-0.87, $P<0.001)$. Dronedarone also significantly reduced the incidence of symptomatic first recurrence $(37.7 \%$ versus $46.0 \% ; P<0.01)$ and was well tolerated: among all the side effects only serum creatinine levels were significantly higher in the dronedarone group $(2.4 \%$ versus $0.2 \%, P=0.004)$.

\section{Overall quality of the evidence}

The risk of bias was low for the random sequence generation procedure as the risk of attrition bias (Figure 2). No information was reported for the other items. The population included can be considered representative of the patients usually referring to internal medicine or cardiology divisions. EURIDIS/ADONIS demonstrates that dronedarone could have rhythm- and rate-control properties in patients with paroxysmal or persistent AF. However, the outcomes of the trial were not clinically relevant. Moreover dronedarone should have been compared with another antiarrhythmic drug instead of placebo. It is unclear what proportion of patients in the two groups had previously received and discontinued a treatment with amiodarone just before the enrollment: considering amiodarone's long half-life, this could be a confounding factor, at least in the initial stages of the trial.

\section{ATHENA}

A Placebo-Controlled, Double-Blind, Parallel Arm Trial to Assess the Efficacy of Dronedarone $400 \mathrm{mg}$ bid for the Prevention of Cardiovascular Hospitalization or Death from Any Cause in Patients with Atrial Fibrillation/Atrial Flutter
(ATHENA) was a phase III trial that evaluated the effect of $400 \mathrm{mg}$ of dronedarone twice a day versus placebo in 4628 patients with paroxysmal or persistent AF or flutter who had additional risk factors for a major cardiovascular event (Tables 2 and 3). ${ }^{22}$ People were excluded if affected by NYHA class IV congestive heart failure and were enrolled while in sinus rhythm (after cardioversion) or in AF or flutter but subsequently cardioverted. During the course of the trial the inclusion criteria were changed due to a mortality rate lower than expected, so that people were required to be aged 75 years or older to be eligible, or aged between 70 and 75 years with at least one of the specified risk factors, while people younger than 70 years of age were excluded.

The primary end point was the first unplanned hospitalization due to cardiovascular events or death from any cause before admission to the hospital and hospitalization was defined as admission with at least an overnight stay in the hospital. Secondary end points were death from any cause, death from cardiovascular cause, and first hospitalization due to cardiovascular events.

The mean follow-up period was 21 months. The primary composite outcome (incidence of hospitalization due to cardiovascular events or death) was significantly lower in patients receiving dronedarone as compared to those receiving placebo (HR 0.76, 95\% CI 0.69-0.84, $P<0.001$; Table 4). This outcome appeared to be mainly driven by a lower risk of hospitalization for cardiovascular events and, in particular, for AF ( 675 cases in the dronedarone group versus 859 in placebo; HR $0.74,95 \%$ CI $0.67-0.82, P<0.001)$. No statistical difference for other secondary end points was found, except for cardiovascular mortality, which was lower in patients receiving dronedarone (HR 0.71, 95\% CI $0.51-0.98, P=0.03)$. Treatment was stopped early in $30.2 \%$ of patients in the dronedarone group as compared with $30.8 \%$ of those in the placebo group (statistical significance not reported). The main reasons for discontinuation were adverse events (290 with dronedarone versus $187, P<0.001$ ): gastrointestinal events, increase in serum creatinine, bradycardia, and QT-interval prolongation were found to be associated with dronedarone (Table 5).

\section{Overall quality of the evidence}

The risk of bias was low for the sequence generation process and the attrition. No information about the other dimension was reported in the paper (Figure 2). ATHENA shows that dronedarone reduces the incidence of hospitalization due to cardiovascular events or death in patients with paroxysmal or persistent AF or flutter who had additional 
risk factors for major cardiovascular events. It is important to underline that the trial has many weaknesses. First of all, the difference in the primary composite end point appears to be only due to one of its components such as the reduction of hospitalization for cardiovascular events. This may have been driven by the reduction in hospitalizations for the recurrence of AF episodes that could have been treated by electrical cardioversion with subsequent early discharge. Considering such a short hospital stay (discharge within 12 hours) as hospitalization may generate a misleading outcome evaluation. For this reason we believe that the study shows only that dronedarone reduced the recurrence of AF. In addition, in the dronedarone group there were significantly more women and this difference was not reported at the time of publication in The New England Journal of Medicine. ${ }^{27}$ For the evaluation of the adverse effects, the length of the follow-up can be considered adequate.

\section{DIONYSOS}

The Efficacy and Safety of Dronedarone Versus Amiodarone for the Maintenance of Sinus Rhythm in Patients With Atrial Fibrillation (DIONYSOS) is a phase III, randomized, double blind trial that evaluated the efficacy and safety of dronedarone (400 $\mathrm{mg}$ twice a day) versus amiodarone $(600 \mathrm{mg}$ /day for 28 days and then $200 \mathrm{mg}$ /day for at least 6 months) for the maintenance of sinus rhythm in patients with documented AF that had lasted more than 72 hours (Tables 2 and 3). ${ }^{23}$ The primary outcome was a composite measure of treatment failure, described as the first recurrence of AF including unsuccessful electrical cardioversion, no spontaneous conversion and no electrical cardioversion or premature drug discontinuation for lack of efficacy or intolerance. The study had also a safety/tolerability end point composed by the occurrence of thyroid-, hepatic-, pulmonary-, neurologic-, skin-, eye or gastrointestinal-specific events, or premature study drug discontinuation for intolerance. Analysis of the safety end point without gastrointestinal events (ie, diarrhea, nausea, and vomiting) was prespecified. The minimum duration of follow-up was 6 months.

Five hundred and four subjects were enrolled and the two treatment groups were well balanced in terms of demographic characteristics, cardiovascular history, and co-therapy assumed, but in the amiodarone group the AF episode ongoing at the moment of the enrolment had a longer duration (54 versus 47.5 days in the dronedarone group, $P=0.0311$ ).

The primary end point at 12 months was in favor of amiodarone (HR 1.59, 95\% CI 1.28-1.98, $P \leq 0.0001$; Table 4). Although not significantly different, the incidence of the main safety end point was reduced in the dronedarone group as compared to amiodarone (HR 0.80, 95\% CI 0.60-1.07, $P=0.129$ ), and there was a statistically significant difference in favor of dronedarone when gastrointestinal events were excluded from the main safety end point (HR 0.61, 95\% CI 0.44-0.84, $P=0.002$; Table 5).

\section{Overall quality of the evidence}

The risk of bias of the study cannot be properly evaluated as much information in the reporting was lacking (Figure 2). DIONYSOS is a relevant trial because it is the only one that compares dronedarone to amiodarone. It demonstrates that the supposed improved tolerability of dronedarone compared to the known poor tolerability of amiodarone does not compensate for its modest antiarrhythmic activity. It must be remarked that the efficacy end points of the study are not hard outcomes. Furthermore, the trial evaluated only combined end points that were not exhaustively described and the differences among the definitions of drug discontinuation for lack of efficacy, intolerance, or due to any adverse event are not clear. The follow-up (6 months) is not long enough to draw relevant information about safety.

\section{RCTs including patients in permanent $A F$ ERATO}

The Efficacy and safety of dRonedArone for The cOntrol of ventricular rate during atrial fibrillation (ERATO) was a phase III, double-blind, randomized controlled trial designed to assess the efficacy of dronedarone for ventricular rate control in patients with permanent AF. The objective was to perform a rate-control strategy. ${ }^{24}$

Subjects with symptomatic permanent AF, for whom cardioversion was appropriate, were enrolled in nine European countries. Patients with NYHA class III or IV congestive heart failure were excluded (Tables 2 and 3). One hundred and eighty-five patients were randomized to receive either dronedarone $400 \mathrm{mg}$ twice a day or placebo for 6 months. The primary end point was the change in mean ventricular rate between baseline and day 14 , as assessed by 24-hour electrocardiogram Holter monitoring. A priori defined subgroup analysis was programmed, stratifying the primary outcome according to concomitant use of other rate-lowering drugs. Secondary end points of interest were the changes in mean ventricular rate during submaximal and maximal exercise between the baseline and day 14 and the changes in maximal exercise duration, as assessed by exercise electrocardiogram test. 
In the dronedarone group there was a significantly greater reduction of mean 24-hour ventricular rate at day 14 ( -11 beats/minute versus +0.7 beats/minute in the placebo group, $P<0.0001)$ and similar results were observed after 4 months (Table 4). The efficacy was of greater magnitude during submaximal and maximal exercise at day 14. The effects of dronedarone rate were additive to those of concomitant rate-controlling drugs. The incidence of adverse events was slightly higher in the dronedarone arm (65 events versus $53,77 \%$ and $60 \%$ ), although serious adverse cardiovascular events were rare and not significantly different between the two groups (Table 5).

\section{Overall quality of the evidence}

The methodology of the trial is not described in detail and consequently we cannot give a proper evaluation of the risk of bias (Figure 2). ERATO shows that dronedarone has rate control effects in patients with permanent AF. The results are coherent to each other and consistent but none of the primary or secondary outcomes can be defined as clinically relevant. Furthermore, the study was not projected to compare dronedarone to traditional rate-control therapies like digoxin, calcium antagonists, or beta blockers, so the comparison used cannot be considered adequate.

\section{PALLAS}

The Permanent Atrial Fibrillation Outcome Study Using Dronedarone on Top of Standard Therapy (PALLAS) was a double-blind phase III study that evaluated the effect of dronedarone (400 mg twice a day) versus placebo on major vascular events or unplanned hospitalization for cardiovascular causes. ${ }^{25}$ The study included patients with permanent AF or flutter at high risk for vascular events (Tables 2 and 3). Patients affected by NYHA class IV congestive heart failure were excluded. The two treatment groups were well balanced for demographic characteristics and cardiovascular history.

The first primary outcome was a composite of stroke, myocardial infarction, systemic embolism, or death from cardiovascular causes. The second primary outcome was a composite of unplanned hospitalization for a cardiovascular cause or death.

Ten thousand, eight hundred patients were estimated to be enrolled during a 2-year period but the study was terminated for safety reasons after 1 year and the randomization of 3236 participants (1619 treated with dronedarone and 1617 in the placebo group). In fact, the first primary outcome occurred in 43 patients receiving dronedarone and in
19 receiving placebo (HR 2.29, 95\% CI 1.34-3.94; $P=0.002$; Table 4). There were more deaths for cardiovascular causes in the dronedarone group (21 versus 10 in the placebo group, HR 2.11, 95\% CI 1.00-4.49; $P=0.046)$, including death from arrhythmia in 13 and four patients in each arm, respectively (HR 3.26, 95\% CI 1.06-10.00; $P=0.03$ ). Twenty-three strokes occurred in the dronedarone group and ten in the placebo group (HR 2.32, 95\% CI 1.11-4.88; $P=0.02$ ). Hospitalization for heart failure was observed in 43 patients in the dronedarone group and 24 in the placebo group (HR $1.81 ; 95 \%$ CI $1.10-2.99 ; P=0.02)$. Unplanned hospitalization for cardiovascular causes occurred in 113 patients receiving dronedarone and 59 patients receiving placebo (HR $1.97,95 \%$ CI 1.44-2.70, $P<0.001)$. Study medication was permanently discontinued prematurely in $21 \%$ of patients treated with dronedarone and in $11 \%$ of those in the placebo group $(P<0.001)$.

\section{Overall quality of the evidence}

There is insufficient information reported in the paper to permit evaluation of the risk of bias (Figure 2). PALLAS considered clinically relevant end points (stroke, myocardial infarction, systemic embolism, or death from cardiovascular causes) and produced the important evidence that dronedarone should not be used in patients with permanent AF or flutter at high risk for vascular events. The results of the study are coherent to each other and consistent.

\section{RCTs including patients with heart failure ANDROMEDA}

The Antiarrhythmic Trial with Dronedarone in Moderate to Severe CHF Evaluating Morbidity Decrease (ANDROMEDA) is a phase III trial that evaluated the use of dronedarone (400 mg twice a day) versus placebo in hospitalized patients with new or worsening heart failure and severe systolic dysfunction heart failure ${ }^{26}$ (Tables 2 and 3). Consecutive adult patients were included.

The trial had a composed primary end point, including death from any cause or hospitalization for worsening heart failure. Secondary end points were death from any cause, hospitalization for worsening heart failure, occurrence of AF or atrial flutter, death from arrhythmia, or sudden death. The overall percentage of participants with a history of $\mathrm{AF} /$ flutter was $38 \%$, while the percentage of subjects in $\mathrm{AF}$ at the baseline was $25 \%$ (23.2\% in the dronedarone and $26.8 \%$ in the placebo group).

A sample size of 1000 patients had been planned, but after the inclusion of 627 patients ( 310 in the dronedarone group 
and 317 in the placebo group), the trial was prematurely stopped for an excess of deaths in patients taking dronedarone (25 versus 12 in the placebo group, HR 2.13, 95\% CI $1.07-4.25, P=0.03)$. The primary composite outcome was in the same direction as mortality but did not reach a statistical difference (HR 1.38, 95\% CI 0.92-2.09; Table 4). Deaths in the dronedarone group were mainly related to progressive heart failure, arrhythmia, or sudden death. No significant difference in mortality between the two groups was observed in the 6-month follow-up after discontinuation of the treatment (HR 1.13, 95\% CI 0.73-1.74, $P=0.60$ ) and no significant difference was reported for other adverse events except for a higher number of patients with increased creatinine levels in the dronedarone group (Table 5).

\section{Overall quality of the evidence}

The risk of bias in the study was low overall but no information about allocation concealment and blinding of outcome assessment was reported (Figure 2). ANDROMEDA is the only trial using hard and clinically relevant end points to test the effect of dronedarone in patients with severe congestive heart failure for whom AF was not an inclusion criterion. Considering that amiodarone is one of the few antiarrhythmic drugs that does not increase mortality in patients with moderate to severe left ventricular dysfunction, the study was projected in the hypothesis that dronedarone would have improved prognosis in these patients. On the contrary, the results show that dronedarone should not be used in patients with severe congestive heart failure, with or without AF.

\section{Discussion}

\section{Summary of evidence}

The major findings have to be defined in the different populations studied in the various RCTs analyzed and are the following: (1) compared to placebo, dronedarone reduces the recurrence of AF leading to a lower incidence of hospitalization in patients with nonpermanent AF; (2) dronedarone is less effective than amiodarone for the prevention of recurrent $\mathrm{AF}$; (3) in patients with heart failure or with permanent $\mathrm{AF}$ and a high risk for cardiovascular events dronedarone is associated with increased mortality.

In the management of AF either a "rhythm-control" or "rate-control" strategy can be chosen; to date the superiority of one strategy over the other has not been demonstrated. ${ }^{9-11 .}$

In the "rhythm-control" option, the goal is to prevent AF recurrences in patients with persistent symptoms despite adequate rate-control. The DAFNE and EURIDIS/ADONIS studies evaluated the efficacy of dronedarone in reducing recurrences in subjects with nonpermanent AF. The trials demonstrated that dronedarone is efficacious when compared to placebo. These results are consistent with those of a recent meta-analysis that showed that antiarrhythmics of different pharmacological classes (class IA, class IC, and class III drugs) were efficacious in controlling the recurrences of AF; these occurred $20 \%$ to $50 \%$ less than in patients not receiving antiarrhythmics. ${ }^{12}$ However, the efficacy of antiarrhythmic drugs is limited, as demonstrated by the fact that $\mathrm{AF}$ still recurred in $42 \%$ to $67 \%$ of treated patients in 1 year. ${ }^{12}$ Similarly, in the EURIDIS/ADONIS trials, the $64 \%$ of subjects treated with dronedarone experienced recurrences of AF.

The ATHENA trial showed that in comparison to placebo, dronedarone resulted in a statistically significant reduction in the primary composite end point of time to first cardiovascular hospitalization or death from any cause in patients with nonpermanent AF. It is important to remark that the primary end point was mainly driven by reduction of $\mathrm{AF}$ recurrences, as were the DAFNE and EURIDIS/ ADONIS results.

In an indirect comparison meta-analysis, Piccini et al evaluated eight trials (four trials evaluating amiodarone versus placebo, four dronedarone versus placebo), finding that amiodarone was superior to dronedarone in the prevention of recurrent AF (OR 0.16, 95\% CI 0.06-0.42, $P<0.001) .{ }^{28}$ These data appear to support the results of the direct comparison in the DIONYSOS trial that demonstrated the superiority of amiodarone over dronedarone in the prevention of $\mathrm{AF}$ recurrences in subjects with nonpermanent AF (OR 0.44, 95\% CI: 0.30-0.64). ${ }^{28}$ Moreover, in a recent meta-analysis of studies of enrolled patients who recovered sinus rhythm after AF, Lafuente-Lafuente et al showed that the most efficacious drug in preventing recurrences of AF was amiodarone and the related number needed to treat to prevent one event in 1 year was three. ${ }^{12}$

Dronedarone was developed to reduce the adverse events associated with antiarrhythmic class III treatment. It was demonstrated that amiodarone had a greater incidence of adverse events leading to discontinuation of therapy than placebo. ${ }^{12,28}$ In the comparative DIONYSOS trial, it is uncertain whether dronedarone is associated with a lower rate of discontinuations than amiodarone, as all the confidence intervals of the direct comparison cross 1 . It must be noted that the safety results of DIONYSOS are difficult to interpret due to the short duration (6 months) of the trial, taking into account that the toxicities of amiodarone may arise after years of chronic therapy. In studies that enrolled patients 
with paroxysmal or persistent AF, mortality was evaluated in the ATHENA trial only. In the latter, cardiovascular mortality was significantly lower in patients receiving dronedarone compared to those receiving placebo; it is difficult to comment on the data taking into account that the mortality was a secondary end point of the study. The results should be confirmed in an ad hoc designed study. The meta-analysis of Lafuente-Lafuente et al demonstrated that mortality was not modified by antiarrhythmic drugs, except for disopyramide and quinidine that were associated with increased mortality. ${ }^{12}$

Considering patients with permanent AF and the choice of "rate-control" therapy, dronedarone was evaluated in the ERATO and PALLAS trials. The ERATO trial showed that dronedarone has rate-control effects in patients with permanent AF; considering the surrogate outcomes of the trials, data were not sufficient to recommend dronedarone for rate control. The PALLAS trial considered clinically relevant outcomes and the study was stopped early since strokes, myocardial infarctions, systemic embolisms, or deaths from cardiovascular causes were significantly more frequent in subjects treated with dronedarone as compared to placebo. These results indicate that dronedarone should not be prescribed in subjects with permanent AF and a high risk of vascular events.

The ANDROMEDA trial intended to study subjects with new symptomatic heart failure (more than NYHA functional class II) and severe left ventricular systolic dysfunction ( $\leq 35 \%$ ); a not insignificant proportion of patients in the study had AF. It was terminated prematurely because of excess mortality among patients in the dronedarone group, demonstrating that the drug should not be used in ANDROMEDAlike patients, with or without $\mathrm{AF}$.

It is difficult to determine the reasons for the excess mortality in PALLAS and in ANDROMEDA trials. The patients included had risk factors for major vascular events, but the risk profile was not considerably different from that observed in ATHENA trial where the results were completely different. Considering the results from PALLAS and ANDROMEDA, safety concerns about dronedarone are increasing. Recently, the US Food and Drug Administration reported cases of hepatocellular liver injury and hepatic failure in patients treated with dronedarone, including two postmarketing reports of acute hepatic failure requiring transplantation. ${ }^{29}$

The clinical practice guidelines provide a summary of the most current literature and recommendations for the prevention and management of conditions based on current scientific evidence. The ACCF/AHA and ESC guidelines do not give identical recommendations for non-permanent $\mathrm{AF}$ management and are dissimilar for the indications about the use of dronedarone (Table 1). Cardiovascular guidelines on the same clinical question by different expert groups often disagree,$^{30}$ and the latter can be a barrier to acceptance of guidelines. However, disagreement among recommendations is not necessarily a sign of poor quality, as weak evidence may lead to discordant conclusions. Conflict of interest can also play a role especially in the cardiovascular field. It has been reported that half of the authors and reviewers of cardiovascular clinical practice guidelines have at least one conflict of interest. ${ }^{31}$ In the ACCF/AHA guidelines committee $4 / 12$ panelists reported a conflict of interest with the manufacturer of dronedarone, although among them three refused to vote on recommendations regarding the use of the drug; ten of 25 authors of ESC guidelines reported conflict of interest with the manufacturer of dronedarone. We believe that disclosure of conflicts of interest allows the professional integrity of guideline group members to be assessed. Moreover, instruments to assess the quality of clinical practice guidelines are pivotal. The Appraisal of Guidelines for Research and Evaluation (AGREE) instrument ${ }^{32}$ provides a validated, internationally agreed framework for evaluating both the quality of the reporting and the quality of some aspects of recommendations. Its application can be the first step toward quality improvement and protection from financial and nonfinancial conflicts of interest.

In the clinical scenario presented, the patient had persistent AF and structural heart disease; the subject could be considered an ATHENA-like patient, but, after the considerations made, we believe that the quality and strength of the evidence for prescribing dronedarone are not sufficient. We would not have prescribed the drug.

\section{Study limitations}

Our analysis was restricted to RCTs. It is well known that these trials minimize bias, but concern about external validity can arise since they may not reflect patients treated in general clinical practice. Furthermore, in many of the included trials, information about the methodology used was not reported (methods of sequence generation and allocation concealment, strategies to guarantee blindness, etc) so we were not able to evaluate their internal quality. The follow-up of the studies was short in duration, while for properly evaluating the toxicity of dronedarone more long-term data are needed. Finally, the manufacturer of dronedarone sponsored all the trials. 


\section{Conclusion}

In subjects with AF, several randomized controlled trials have evaluated the efficacy and the safety of dronedarone versus placebo and one compared dronedarone with amiodarone. The results of the studies suggest that dronedarone should be avoided in patients with permanent AF and a high risk for cardiovascular events or severe congestive heart failure. In subjects with paroxysmal or persistent $\mathrm{AF}$, dronedarone, like most antiarrhythmic drugs, reduces the recurrence of AF compared to placebo but relapse rates in the first year of therapy are high. Furthermore, dronedarone showed to be less effective than amiodarone. Considering the data derived from the trials that were terminated prematurely because of safety concerns and the absence of studies showing the superiority of dronedarone over the other antarrhythmics, further investigations are needed before supporting the use of dronedarone for the maintainance of sinus rhythm in patients with atrial fibrillation.

\section{Disclosure}

The authors report no conflicts of interest.

\section{References}

1. Lip GY, Nieuwlaat R, Pisters R, Lane DA, Crijns HJ. Refining clinical risk stratification for predicting stroke and thromboembolism in atrial fibrillation using a novel risk factor-based approach: the Euro Heart Survey on Atrial Fibrillation. Chest. 2010;137(2):263-272.

2. Wann LS, Curtis AB, January CT, et al. 2011 ACCF/AHA/HRS focused update on the management of patients with atrial fibrillation (Updating the 2006 Guideline): a report of the American College of Cardiology Foundation/American Heart Association Task Force on Practice Guidelines. J Am Coll Cardiol. 2011;57(2):223-242.

3. European Heart Rhythm Association; Heart Rhythm Society, Fuster V, Rydén LE, Cannom DS, et al. ACC/AHA/ESC 2006 guidelines for the management of patients with atrial fibrillation - executive summary: a report of the American College of Cardiology/American Heart Association Task Force on Practice Guidelines and the European Society of Cardiology Committee for Practice Guidelines (Writing Committee to Revise the 2001 Guidelines for the Management of Patients With Atrial Fibrillation). J Am Coll Cardiol. 2006;48(4):854-906.

4. European Heart Rhythm Association, European Association for CardioThoracic Surgery; Camm AJ, Kirchhof P, Lip GY, et al. Guidelines for the management of atrial fibrillation: the Task Force for the Management of Atrial Fibrillation of the European Society of Cardiology (ESC). Eur Heart J. 2010;31(19):2369-2429.

5. Go AS, Hylek EM, Phillips KA, et al. Prevalence of diagnosed atrial fibrillation in adults: national implications for rhythm management and stroke prevention: the AnTicoagulation and Risk Factors in Atrial Fibrillation (ATRIA) Study. JAMA. 2001;285(18):2370-2375.

6. Ruigomez A, Johansson S, Wallander MA, Rodriguez LA. Incidence of chronic atrial fibrillation in general practice and its treatment pattern. $J$ Clin Epidemiol. 2002;55(4):358-363.

7. Krahn AD, Manfreda J, Tate RB, Mathewson FA, Cuddy TE. The natural history of atrial fibrillation: incidence, risk factors, and prognosis in the Manitoba Follow-Up Study. Am J Med. 1995;98(5):476-484.

8. Stewart S, Hart CL, Hole DJ, McMurray JJ. A population-based study of the long-term risks associated with atrial fibrillation: 20-year follow-up of the Renfrew/Paisley study. Am J Med. 2002;113(5):359-364.
9. Cordina J, Mead G. Pharmacological cardioversion for atrial fibrillation and flutter. Cochrane Database Syst Rev. 2005(2):CD003713.

10. De Denus S, Sanoski CA, Carlsson J, Opolski G, Spinler SA. Rate vs rhythm control in patients with atrial fibrillation: a meta-analysis. Arch Intern Med. 2005;165(3):258-262.

11. Testa L, Biondi-Zoccai GG, Dello Russo A, Bellocci F, Andreotti F, Crea F. Rate-control vs rhythm-control in patients with atrial fibrillation: a meta-analysis. Eur Heart J. 2005;26(19):2000-2006.

12. Lafuente-Lafuente C, Mouly S, Longas-Tejero MA, Bergmann JF. Antiarrhythmics for maintaining sinus rhythm after cardioversion of atrial fibrillation. Cochrane Database Syst Rev. 2007(4): CD005049.

13. AFFIRM First Antiarrhythmic Drug Substudy Investigators. Maintenance of sinus rhythm in patients with atrial fibrillation: an AFFIRM substudy of the first antiarrhythmic drug. J Am Coll Cardiol. 2003;42(1):20-29.

14. Zimetbaum P. Amiodarone for atrial fibrillation. $N$ Engl J Med. 2007; 356(9):935-941.

15. Liberati A, Altman DG, Tetzlaff J, et al. The PRISMA statement for reporting systematic reviews and meta-analyses of studies that evaluate health care interventions: explanation and elaboration. Ann Intern Med. 2009;151(4):W65-W94.

16. Sackett DL, Haynes RB, Guyatt GH, Tugwell P. Clinical Epidemiology, A Basic Science for Clinical Medicine. 2nd ed. Boston: Little, Brown; 1991.

17. Higgins JPT, Altman DG, Sterne, JAC, editors. Chapter 8: Assessing risk of bias in included studies. In: Higgins JPT, Green S, editors Cochrane Handbook for Systematic Reviews of Interventions. Version 5.1.0 [updated March 2011]. The Cochrane Collaboration, 2011. Available from http://www.cochrane-handbook.org.

18. Guyatt GH, Oxman AD, Kunz R, et al; for the GRADE Working Group. GRADE guidelines: 8. Rating the quality of evidence - indirectness. J Clin Epidemiol. 2011;64(12):1303-1310.

19. Rothwell PM. Factors that can affect the external validity of randomised controlled trials. PLoS Clin Trials. 2006;1(1):e9.

20. Touboul P, Brugada J, Capucci A, Crijns HJ, Edvardsson N, Hohnloser SH. Dronedarone for prevention of atrial fibrillation: a dose-ranging study. Eur Heart J. 2003;24(16):1481-1487.

21. Singh BN, Connolly SJ, Crijns HJ, et al. Dronedarone for maintenance of sinus rhythm in atrial fibrillation or flutter. $N$ Engl J Med. 2007; 357(10):987-999.

22. Hohnloser SH, Crijns HJ, van Eickels M, et al; for the ATHENA Investigators. Effect of dronedarone on cardiovascular events in atrial fibrillation. N Engl J Med. 2009;360(7):668-678.

23. Le Heuzey JY, De Ferrari GM, Radzik D, Santini M, Zhu J, Davy JM. A short-term, randomized, double-blind, parallel-group study to evaluate the efficacy and safety of dronedarone versus amiodarone in patients with persistent atrial fibrillation: the DIONYSOS study. J Cardiovasc Electrophysiol. 2010;21(6):597-605.

24. Davy JM, Herold M, Hoglund C; et al for the ERATO Study Investigators. Dronedarone for the control of ventricular rate in permanent atrial fibrillation: the Efficacy and safety of dRonedArone for the cOntrol of ventricular rate during atrial fibrillation (ERATO) study. Am Heart J. 2008;156(3):527. e521-e529.

25. Connolly SJ, Camm AJ, Halperin JL; et al for the PALLAS Investigators. Dronedarone in high-risk permanent atrial fibrillation. $N$ Engl $J$ Med. 2011;365(24):2268-2276.

26. Kober L, Torp-Pedersen C, McMurray JJ; et al for the Dronedarone Study Group. Increased mortality after dronedarone therapy for severe heart failure. $N$ Engl J Med. 2008;358(25):2678-2687.

27. Pugliano M, Costantino G, Podda GM; for the ATHENA Investigators. Dronedarone in atrial fibrillation. $N$ Engl J Med. 2009;360(23): 2479-2480; author reply 2480-2471.

28. Piccini JP, Hasselblad V, Peterson ED, Washam JB, Califf RM, Kong DF. Comparative efficacy of dronedarone and amiodarone for the maintenance of sinus rhythm in patients with atrial fibrillation. J Am Coll Cardiol. 2009;54(12):1089-1095. 
29. http://www.fda.com [homepage on the Internet] United States Food and Drug Administration. FDA Drug Safety Communication January 20, 2011. Severe liver injury associated with the use of dronedarone (marketed as Multaq). Available from: http://www.fda.gov/Drugs/ DrugSafety/ucm240011.htm. Accessed March 28, 2012.

30. Ferket BS, Colkesen EB, Visser JJ, et al. Systematic review of guidelines on cardiovascular risk assessment: Which recommendations should clinicians follow for a cardiovascular health check? Arch Intern Med. 2010;170(1):27-40.
31. Mendelson TB, Meltzer M, Campbell EG, Caplan AL, Kirkpatrick JN. Conflicts of interest in cardiovascular clinical practice guidelines. Arch Intern Med. 2011;171(6):577-584.

32. The Appraisal of Guidelines for Research and Evaluation instrument. Available for download from: http://www.agreetrust.org. Accessed March 29, 2012.

\section{Publish your work in this journal}

The International Journal of General Medicine is an international, peer-reviewed open-access journal that focuses on general and internal medicine, pathogenesis, epidemiology, diagnosis, monitoring and treatment protocols. The journal is characterized by the rapid reporting of reviews, original research and clinical studies across all disease areas.
A key focus is the elucidation of disease processes and management protocols resulting in improved outcomes for the patient.The manuscript management system is completely online and includes a very quick and fair peer-review system. Visit http://www.dovepress.com/ testimonials.php to read real quotes from published authors.

Submit your manuscript here: http://www.dovepress.com/international-journal-of-general-medicine-journal 